\title{
Primary sternal tuberculosis mimicking a lytic bone tumor lesion
}

\author{
Elisa Calabrò', Ugo Pastorino² \\ 1 Division of Internal Medicine, ASST Fatebenefratelli Sacco, Milan \\ 2 Division of Thoracic Surgery, Fondazione IRCCS Istituto Nazionale dei Tumori, Milan, Italy
}

\begin{abstract}
Tubercular sternal osteomyelitis is a rare manifestation of tuberculosis. We describe the case of a 51-years-old male patient, presenting with three months history of pain and swelling of the central chest wall, referred to our hospital with clinical diagnosis of chondrosarcoma of the sternum.
\end{abstract}

\section{Introduction}

Extrapulmonary form of tuberculosis involving the sternum is quite rare. We describe a case of male patient with isolated sternal osteomyelitis with no extrasternal tuberculosis.

\section{Case Report}

A never smoker 51-year-old Pakistan man, who was living in Italy for 20 years, was admitted to another hospital for evaluation of a pain of the upper chest wall that had been present for 3 months. During admission, patient underwent chest CT scan that revealed an osteolytic lesion of the sternum with cranial-caudal extension of $3 \mathrm{~cm}$ and maximum diameter of $2.8 \mathrm{~cm}$, no parenchymal lesions were detected. Positron Emission Tomography scan revealed increased sternal lesion FDG uptake with 15 of SUV max. Nuclear Magnetic Resonance showed

Corresponding author: Dr. Elisa Calabrò, Division of Internal Medicine, ASST Fatebenefratelli Sacco, Corso di Porta Nuova, 20121 Milano, Italy. Tel. +39.02.63632568 - Fax +39.02.63632243.

E-mail: elisa.calabro@asst-fbf-sacco.it

Key words: Sternal tuberculosis; Mycobacterium tuberculosis.

Conflict of interest: The authors declares that there is no conflict of interest regarding the publication of this article.

Received for publication: 25 March 2018

Accepted for publication: 9 May 2018

(C) Copyright E. Calabrò and U. Pastorino, 2018

Tipografia PI-ME Editrice, Italy

Monaldi Archives for Chest Disease 2018; 88:931

doi: 10.4081/monaldi.2018.931

This article is distributed under the terms of the Creative Commons Attribution Noncommercial License (by-nc 4.0) which permits any noncommercial use, distribution, and reproduction in any medium, provided the original author(s) and source are credited. presence of sternal expansive lesion, with transverse diameter of $5 \mathrm{~cm}$, anteroposterior of $3 \mathrm{~cm}$ and craniocaudal extension of $4 \mathrm{~cm}$, the findings were suggestive for chondrosarcoma. After one month the patient was admitted to the Istituto Nazionale Tumori of Milan for further diagnostic evaluation.

Physical examination at admission revealed satisfactory general health, and no fever. The head and neck examination were normal without lymphadenopathy. Examination of the chest wall demonstrated sternal swelling of about $5 \mathrm{~cm}$, spontaneously painful, with poorly defined margins. Arterial blood analysis on air and respiratory function tests were normal. The WBC was $8.40 \times 10^{3} / \mu \mathrm{L}$, serum tests for HIV, hepatitis B and C were negative, C-Reactive Proteine was $35 \mathrm{mg} / \mathrm{L}$.

The CT scans showed a solid lesion, destructing the sternal body, with maximal antero-posterior extension of $4 \mathrm{~cm}$ and central hypodense areas of necrosis (Figures 1 and 2). CT images with central hypodense areas of necrosis (Figure 3). Calcifications were present within the lesion. Tru-Cut biopsy revealed granulomatous inflammation containing giant cells with absence of caseous necrosis, no evidence of malignancy. A PPD test was positive with $19 \mathrm{~mm}$ of max diameter induration. QuantiFERON-TB Gold tests confirmed a cell-mediated immune reactivity to Mycobacterium tuberculosis. Three sputum samples were negative for acid fast bacilli. Three consecutive sternal fine needle aspirations were performed and histological examination demonstrated chronic necrotizing granulomatous inflammation involving the bone but no evidence of malignancy or acid-fast bacilli. After two weeks, cultures identified Mycobacterium tuberculosis, and the patient was referred to the National Tuberculosis Center in Milan and started on isoniazid, pyrazinemide, rifampin and ethambutol therapy. Contacts and relatives were referred to the same Center for screening, prevention and surveillance. After two months of treatment, the sternal swelling had reduced in size and his symptoms had disappeared, the patient continued therapy for six months achieving a complete clinical recovery.

\section{Discussion}

Bone involvement accounts for 1-3\% of tuberculosis and sporadic cases of sternal lesions have been reported in literature (less than 1\%) [1-5]. Sternal tuberculosis is characterized by painful swelling, fever, local erythema and aspecific clinical symptomatology. It usually occurs in young subjects living in areas where tuberculosis is endemic, without systemic disease manifestation [2-5]. In this study we describe a case of male patients referred to our Department for sternal swelling of about $5 \mathrm{~cm}$, spontaneously painful, with poorly defined margins.

Sternal tuberculosis involvement cannot be discriminated from other infective lesions or primary bone tumors, especially from chondrosarcoma. Because of the similarities in the clinical presen- 


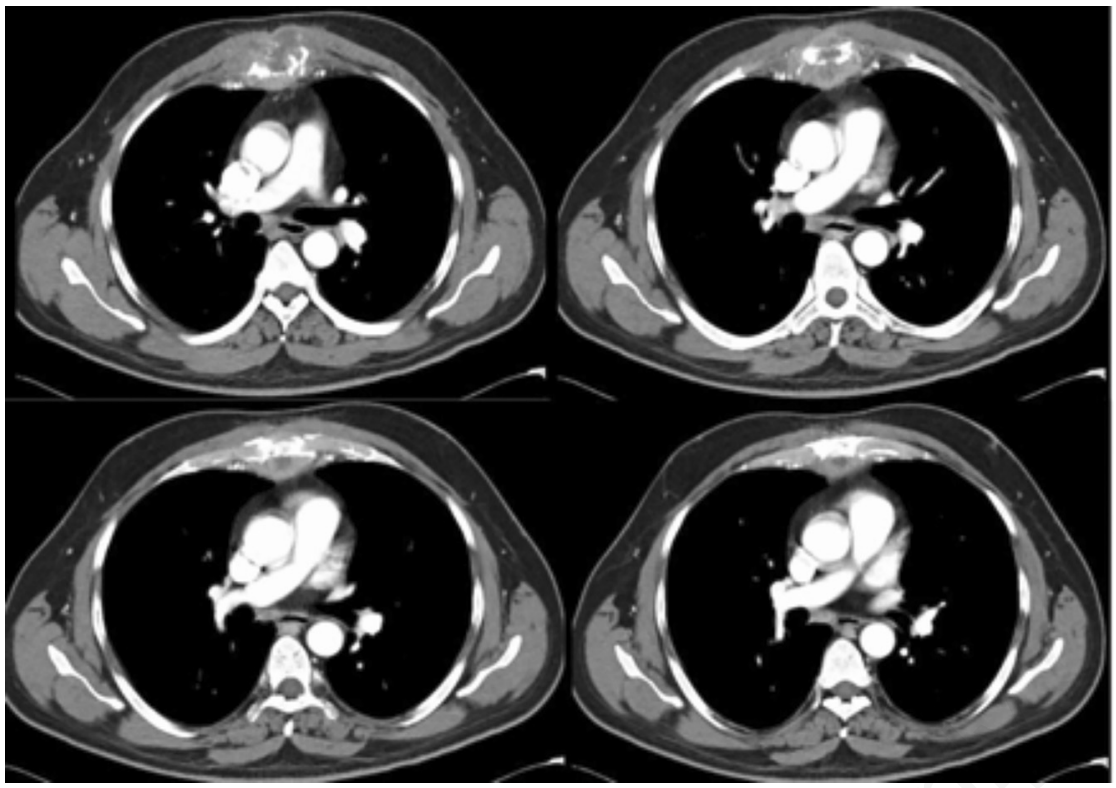

Figure 1. Transverse contrast enhanced CT with extensive lytic lesion of the sternum associated with a soft tissue mass.
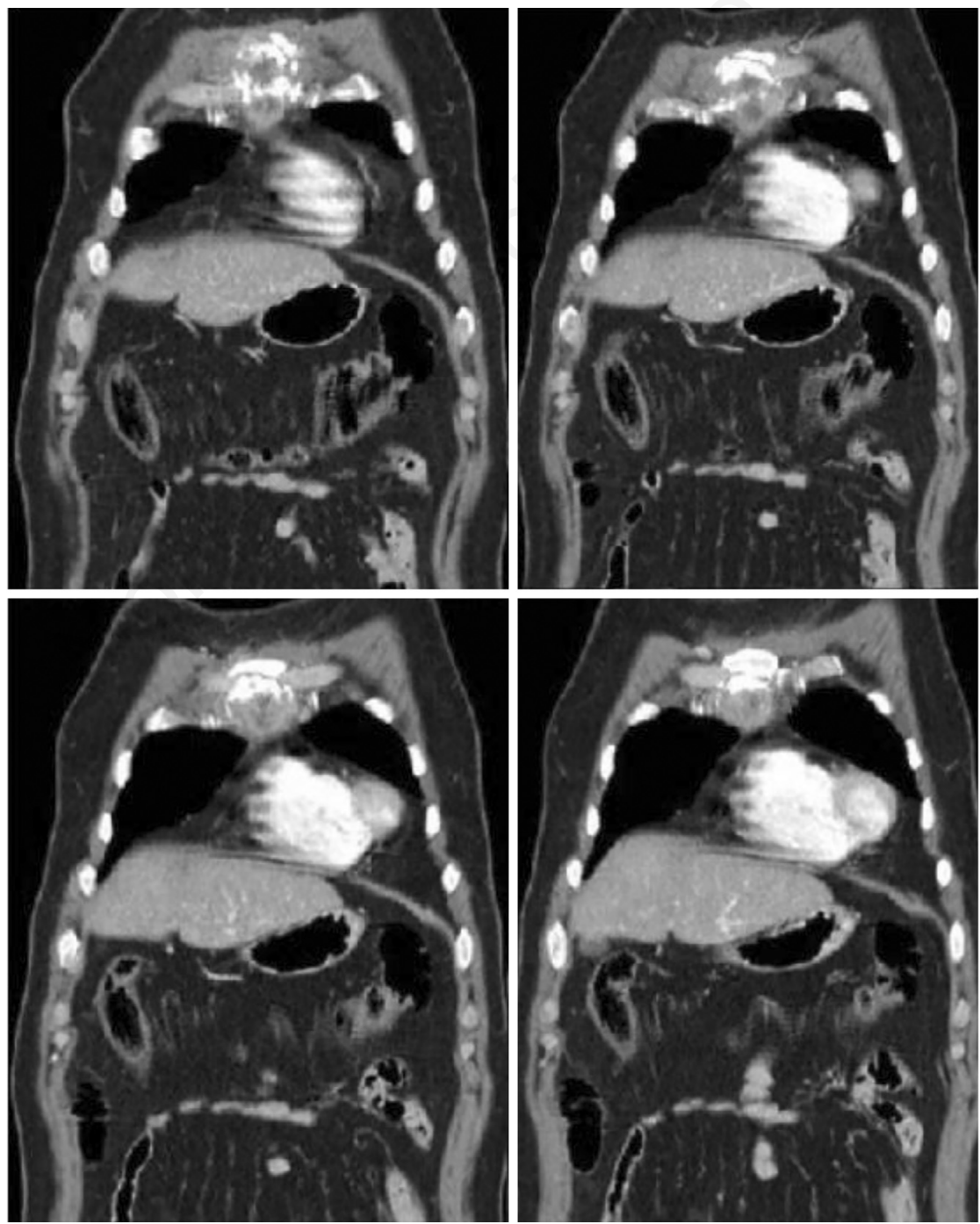

Figure 2. Coronal volume-rendered reconstruction during same imaging phase CT confirm osteolytic lesion of the sternum. 

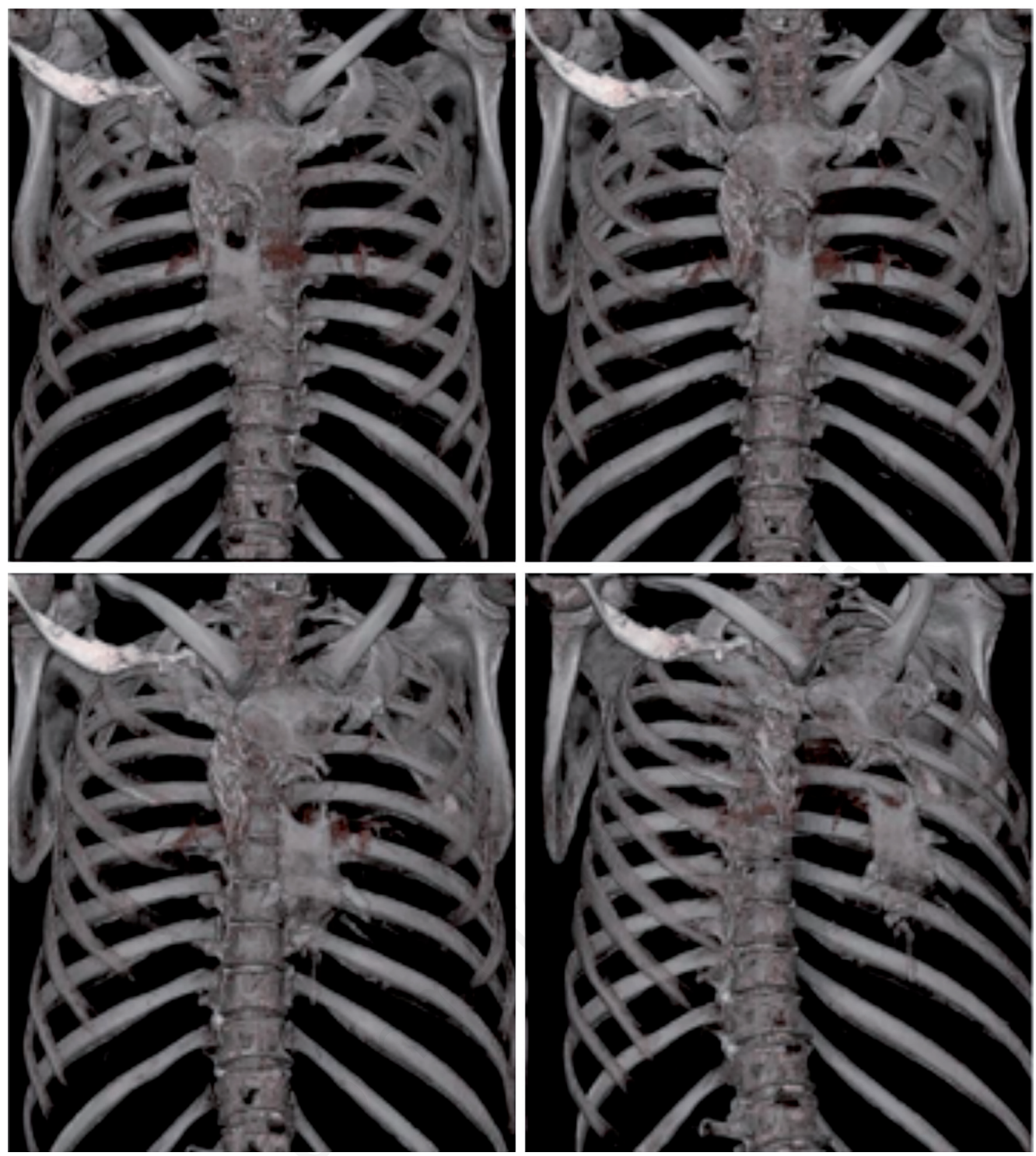

Figure 3. 3D-CT reconstruction with osteolytic lesion of the sternum.

tation with a great range of diseases, diagnosis was made with considerable delay in this patient. CT and magnetic resonance imaging are helpful for diagnosis, but the definitive diagnosis of sternal tuberculosis is based on microbiological and histopathological confirmation. There is no consensus on the optimal therapy and treatment options depend on the extent of disease. In the most reported cases, as in our patient, medical therapy alone was used with a cure rates approaching 95\%.

Antibacterial treatment without unequivocal diagnosis is risky in patients originating from endemic areas such as Pakistan, since resistant forms of tuberculosis are very common, and effective therapy requires the combination of 4 to 5 drugs, which may be very toxic on long-term administration.

\section{Conclusions}

This case illustrates the need to maintain a high index of suspicion and to consider tubercular infection a potential differential diagnosis in bone lesion. In fact, early diagnosis and treatment are very important to avoid the progression towards irreversible damage and to ensure a prompt recovery.

\section{References}

1. Rizzo V, Salmasi Y, Hunter M, Sidhu P. Delayed diagnosis of chronic postoperative sternal infection: a rare case of sternal tuberculosis. BMJ Case Rep 2018; doi:10.1136/bcr-2017-223650.

2. de Carli DM, Severo MD, Haygert CJ, et al. Sternal osteomyelitis caused by infection with Mycobacterium tuberculosis. J Bras Pneumol 2009;35:709-12.

3. Bhatia VY, Aggarwal V, Sharma U, et al. Primary tuberculous sternal osteomyelitis: a clinical rarity. Asian Cardiovasc Thorac Ann 2009;17:310-2.

4. Saifudheen K, Anoop TM, Mini PN, et al. Primary tubercular osteomyelitis of the sternum. Int J Infect Dis 2010;14:e164-6.

5. Zhao X, Chen S, Deanda A, Kiev J. A rare presentation of tuberculosis. Am Surg 2006;72;96-7. 